\title{
Enhanced single-particle brightness and photostability of semiconductor polymer dots by enzymatic oxygen scavenging system
}

Zhihe Liu ${ }^{\mathrm{a}}$, Yingkun Yang ${ }^{\mathrm{a}}$, Zezhou Sun ${ }^{\mathrm{a}}$, and Changfeng Wu${ }^{\mathrm{a}, *}$

${ }^{a}$ State Key Laboratory on Integrated Optoelectronics, College of Electronic Science and Engineering, Jilin University, Changchun, Jilin, 130012, China

*Corresponding Author: cwu@jlu.edu.cn

Keywords: semiconductor polymer dots, single-particle imaging, fluorescence microscopy, oxygen scavenging system, photostability

\begin{abstract}
Semiconductor polymer dots (Pdots) are emerging as an excellent fluorescent probe in biology and medicine. However, the photostability of Pdots can't meet the requirements of long term single-particle imaging and tracking applications. Here we describe the enhanced single-particle brightness and photostability of Pdots by using an efficient enzymatic oxygen scavenging system (OSS). Pdots with particle diameters of $21 \mathrm{~nm}$ and $43 \mathrm{~nm}$ (PFBT21 and PFBT43) were prepared by a nanoprecipitation method. Single-particle imaging and photobleaching were performed to investigate the effect of OSS on the per-particle brightness and photostability of Pdots. Our results indicate that the single-particle brightness of the PFBT21 Pdots in OSS was enhanced nearly two times as compare to the PFBT21 Pdots in water. The photobleaching percentages of PFBT21 and PFBT43 in OSS were determined to be $29 \%$ and $33 \%$, respectively. These values are decreased by 2-3 times as compared to those of the same Pdots in water, indicating the significantly improved photostability of Pdots by OSS. This study provides a promising approach for enhancing photostability of Pdots in long term single-particle tracking.
\end{abstract}

\section{Introduction}

Single-particle fluorescence techniques have been widely used in biomedical engineering and biophotonics for investigation of cellular processes such as molecular transport, membrane dynamics, protein folding, and enzyme kinetics [1-8]. Optical super-resolution imaging modalities have been able to resolve subcellular structures and dynamic movements at the accuracy of the nanometer scale $[9,10]$. Bright and photostable probes are essential to achieve precise localization and high resolution in these optical imaging and tracking 
techniques. Commonly, fluorescent proteins [11,12], small-molecule dyes [13], and inorganic quantum dots (Qdots) [14], have been used as fluorescence probes in super-resolution imaging and single-particle fluorescence techniques. In many cases, however, these probes are not bright and stable enough for photon-starved applications such as long-term imaging and single-particle tracking experiments. The fluorescent dyes and proteins suffer from poor photostability, and the intrinsic toxicity of Qdots restrict their widespread applications in biology.

In past years, we and other groups demonstrated semiconductor polymer dots (Pdots) as bright probes for biology and medicine [15-20]. The large absorption sections, high quantum yield, and efficient energy transfer properties make them suitable for biological imaging and sensing applications. In a pioneer single-particle tracking study, the high fluorescence brightness of Pdots yielded a theoretical particle tracking uncertainty of less than $1 \mathrm{~nm}$ on the basis of the trajectories of fixed and freely diffusing particles [21]. We recently investigated the size-dependent single-particle brightness and photobleaching behavior of Pdots and found that the large Pdots (>20 nm) commonly displayed relatively fast photobleaching components and small Pdots $(10 \mathrm{~nm})$ exhibited intensity blinking behaviors [22]. The fast photobleaching significantly diminish the photon number generated from the Pdots, thus limit their application for long-term imaging and tracking studies. Photobleaching is observed in most fluorescent semiconductor polymers in both solution and solid state. One primary pathway for photobleaching is the photooxidation of semiconductor polymer by molecular oxygen [23]. Under light excitation, the formation of singlet oxygen, peroxides, and other reactive oxygen species (ROS) can lead to oxidative damage to the semiconductor polymer backbone. Moreover, the photo-oxidized defects or photogenerated hole polarons can act as highly efficient fluorescence quenchers that significantly exacerbate the photobleaching processes. For small-molecule, intra-molecular triplet energy transfer mechanism is a general approach for improving performance and photostability of organic fluorophores. By restoring the triplet state to ground state through intra-molecular triplet energy transfer, the methods improved the photostability of cyanine fluorophores [24]. In a recent report, photobleaching of fluorescence Pdots was reduced by doping the Pdots with hydrophobic antifade additives that improve the photostability via mechanisms including quenching of the relevant excited electronic states (e.g., triplet fluorophores and singlet oxygen) and scavenging of ROS [25]. However, fast photobleaching components were still observed in the ensemble (bulk) and single-particle photobleaching measurements. 
Here, we describe the enhancement of single-particle brightness and photostability of Pdots by an enzymatic oxygen scavenging system (OSS). The enzymatic OSS was based on the rapid reaction of the glucose and the glucose oxidase that consumed the molecule oxygen $\left.\underline{(O}_{2}\right)$ around the Pdots. Under the same excitation and collection conditions, the single-particle fluorescence brightness of Pdots by using the OSS exhibit a two-fold increase as compared to that of the Pdots in typical air-saturated solutions. Moreover, we performed single-particle photobleaching statistics to estimate the photostability of Pdots in the presence of the OSS. The photostability of Pdots was significantly improved as compared to the Pdots without the OSS. Furthermore, the fast bleaching component that restricts the applications of Pdots in long term single-particle tracking were suppressed by the rapid and efficient enzymatic oxygen scavenging system.

\section{Material and Methods}

\subsection{Materials}

The semiconductor polymer used in this study is poly [(9, 9-dioctylfluorenyl-2, 7-diyl)-co(1, 4-benzo- $\{2,1$ ', 3 $\}$-thiadiazole)] (PFBT, MW=115,000, polydispersity=3.2), purchased from ADS Dyes, Inc. (Quebec, Canada). The solvent tetrahydrofuran (THF, anhydrous, 99.9\%), the functional polymer poly (styrene co maleic anhydride) (PSMA, average $\mathrm{MW}=\sim 1,700)$, glucose (MW=180.16), glucose oxidase (GOx) (MW=160KDa) were purchased from Sigma-Aldrich. All chemical were used without further purification and all experiments were performed at room temperature.

\subsection{Preparation and characterization of semiconductor polymer dots}

The PFBT Pdots were prepared by a nanoprecipitation procedure modified from the previous reports [26,27]. Briefly, the semiconductor polymer PFBT and functional polymer PSMA were dissolved in THF to obtain a concentration of $1 \mathrm{mg} \mathrm{mL}^{-1}$ primitive solution, respectively. As the precursor solution, the PFBT and PSMA primitive solutions were mixed and diluted to $100 \mu \mathrm{g} \mathrm{mL}^{-1}$ and $20 \mu \mathrm{g} \mathrm{mL}^{-1}$, respectively. A $3 \mathrm{~mL}$ quantity of the precursor solution was quickly injected into $10 \mathrm{~mL}$ deionized water under vigorous ultrasonic. The particle size and morphology of the PFBT Pdots were characterized by dynamic light scattering (DLS) and transmission electron microscopy (TEM). DLS measurements were performed using a $1 \mathrm{~cm}$ disposable polystyrene cuvette at $25^{\circ} \mathrm{C}$ with a Malvern Nano ZS 
instrument. Samples for TEM measurements were prepared by drop casting the Pdots dispersion onto copper grids. TEM images were obtained using a Hitachi H-600 microscope operated at $100 \mathrm{KV}$.

\subsection{Single-particle imaging setup}

Single-particle fluorescence imaging was performed on a custom-built total internal reflection fluorescence microscope (TIRF-M). A $488 \mathrm{~nm}$ Sapphire laser beam (Coherent, USA) was directed into a microscope (Olympus, IX71, Japan). A TIRF objective (1.49 NA. UAPON 100X) was used for illumination and fluorescent light collection. Inside the microscope, fluorescence signal was filtered by a $500 \mathrm{~nm}$ long pass filter and imaged on an Andor iXon3 deep-cooled, back-illuminated frame transfer EMCCD (Andor Technology, UK). Fluorescence intensity emitted per particle in a given frame was estimated by integrating the CCD counts over the fluorescence spot.

\subsection{Preparation of oxygen scavenging system}

To prepare the oxygen scavenging system, PFBT Pdots was diluted in deionized water, dried on a cleaned glass bottom petri dish (NEST China). Then the petri dish was filled with deionized water containing $100 \mathrm{nM}$ glucose oxidase $(\mathrm{GOx})(\sim 4.5 \mathrm{~mL})$, then $200 \mu \mathrm{L}$ glucose in deionized water solution $(20 \mathrm{mM})$ was added into the petri dish followed by a rapid encapsulation. Pure deionized water was filled in another petri dish as the control group for comparison.

\section{Results and discussion}

\subsection{Characterizations of semiconductor polymer dots}

We chose the PFBT polymer (Figure 1(a)) in this study because a number of singleparticle imaging and tracking studies demonstrated high brightness and fast emission rate of PFBT Pdots [28-30]. The preparation conditions were optimized to obtain PFBT Pdots of different sizes as the molecular weight and backbone rigidity affect the particles sizes. We obtained small PFBT Pdots (21 nm diameter) using the precursor PFBT polymer in the pure THF solution. Large PFBT Pdots (43 nm diameter) was prepared using the same protocol but increasing water fraction in precursor solutions. The particle size for the Pdots was determined by DLS measurements, as shown in Figure 1(b). We named the PFBT Pdots as 
PFBT21 and PFBT43 according to the average size distribution. The particle size and morphology were further characterized by transmission electron microscopy (TEM). Figure 1(c) shows a typical TEM image of small PFBT Pdots, indicating a spherical morphology. Figure 1(d) shows the absorption and the emission spectrum of PFBT Pdots, indicating a blue absorption band that can be conveniently excited by a 488nm laser. The PFBT Pdots exhibit a green-yellow emission with a fluorescence quantum yield of $\sim 0.30$, as measured by a QE2100 instrument (Otsuka Electronics Co., Ltd., Japan.). Both the particle morphology and fluorescence properties are consistent with the results in previous reports. [30]

\subsection{Single-particle imaging brightness of semiconductor polymer dots}

We investigate the single-particle fluorescence properties of Pdots by scavenging the molecular oxygen $\left(\mathrm{O}_{2}\right)$ in the solution. The enzymatic oxygen scavenging system was constructed by the oxygen consumption reaction of glucose catalyzed by glucose oxidase [3133]. Single-particle imaging and brightness intensity statistics were performed to assess the fluorescence brightness of Pdots in the presence of oxygen scavenging system. As shown in Figure 2(a) and Figure 2(c), PFBT21 show bright diffraction limited fluorescence spots with a high signal to noise ratio (SNR), indicating these small nanoparticles are well suitable for single-particle imaging and tracking studies. The single-particle imaging brightness of per particle was calculated by integrating the EMCCD counts over the fluorescence spot. Several hundreds of PFBT Pdots were analyzed to evaluate the single-particle fluorescence brightness. Figure 2 (b) and Figure 2 (d) presented the intensity histograms of the PFBT Pdots in OSS and water, respectively. The distribution of the brightness histograms can be well fitted by lognormal functions, which indicated that the average brightness (39452 counts) of the PFBT Pdots in OSS is nearly two-fold higher than that in water (20104 counts). The average brightness values of the Pdot particles are consistent with the single-particle fluorescence images (Figure 2(a)), which indicate that the Pdots in the OSS show a higher SNR as compared to those in water (Figure 2(c)). The improvement of single-particle imaging brightness is likely due to the fact that the OSS significantly suppresses the fast photobleaching component observed in Pdots. For single-particle imaging, an exposure time of $100 \mathrm{~ms}$ was employed. It took a couple of seconds to change the field of view to collect each image. During the imaging process, the PFBT Pdots in absence of OSS can be photobleached to some extent. In contrast, the PFBT Pdots in presence of OSS maintain the initial brightness as the OSS can suppress the fast photobleaching. 


\subsection{Photostability of semiconductor polymer dots in Oxygen Scavenging System}

We performed long term tracking experiments to investigate the effect of OSS on the Pdot photostability. In our experiments, single-particle fluorescence intensities of PFBT Pdots were recorded for 120 seconds under continuous excitation. Figure 3(a) and 3(b) show five typical photobleaching trajectories of PFBT21 Pdots in water and OSS, respectively. As seen from Figure 3(a), the PFBT21 in water exhibited obviously fast photobleaching, which is likely due to the presence of photooxdiation induced defects or hole polarons which act as highly efficient fluorescence quenchers [25,34]. In contrast, PFBT21 Pdots in OSS show enhanced photostability and maintained relatively stable fluorescence intensity during the imaging process (Figure 3(b)). It is worth noting the single-particle fluorescence in OSS exhibit some sparse blinking behaviors which was not observed for the same Pdots in absence of OSS. The enzymatic OSS was based on the rapid reaction of the glucose and the GOx which can consume the molecule oxygen $\left(\mathrm{O}_{2}\right)$ around the PFBT nanoparticles. The sparse blinking behavior may be attributable to the triplet state of the semiconductor polymer, a wellknown factor accountable for blinking behavior in single dye molecules. The molecular oxygen $\left(\mathrm{O}_{2}\right)$ can act as the triplet quencher [35]. As molecular oxygen is removed from solution, the triplet state lifetime increases as compared to the Pdot without OSS, thus distinct blinking behaviors can be seen in single molecule time trajectories [36,37]. The OSS enhanced photostability of Pdots is clearly evidenced by a time-lapsed single-particle imaging. As illustrated in Figure 3(c), the fluorescence intensity of Pdots in water rapidly decreased, and the Pdots were hardly detected after 120-second continuous excitation. In contrast, the Pdots in OSS show comparable fluorescence signal in the first frame, but significantly improved fluorescence signal in subsequent frames as compared to those in water. The distinct photobleaching trajectories together with the time-lapsed single-particle imaging unambiguously confirmed the enhanced photostability of Pdots by OSS.

To quantify the photostability, we define a photobleaching percentage within a given time course as described in Equation (1).

$$
\text { PhotobleachingPercentage }=\frac{F_{0}-F}{F_{0}} \times 100 \%
$$

$\mathrm{F}_{0}$ and $\mathrm{F}$ are the initial and final fluorescence intensity in a single-particle photobleaching trajectory, respectively. In our experiment, $\mathrm{F}_{0}$ and $\mathrm{F}$ are the average intensity of the initial and fianl ten frames to eliminate the noise fluctuation, respectively. From this equation, low 
photobleaching percentage after a period of light excitation indicates a high photostability of Pdots. We analyzed a large number of PFBT21 Pdots to obtain the distribution histograms of photobleaching percentage (Figure 3(d)). A lognormal function was then fit to the distribution histogram to determine the average photobleaching percentage of PFBT21 in water and OSS, respectively. As shown in Figure 3(d), the average photobleaching percentage of PFBT21 in water was determined to be $\sim 86 \%$, while the value for Pdots in OSS was $\sim 29 \%$, which indicate the average photostability in OSS was enhanced by about three times as compared to the Pdots in water (Figure 3(d)). The apparent reduction in photobleaching percentage confirmed that the OSS effectively improved the photostability and suppressed the rapid bleaching component of Pdots.

We further investigated the effect of OSS on single-particle photostability of large sized Pdots. Figure 4(a) and 4(b) show five typical photostability trajectories of PFBT43 Pdots in water and OSS, respectively. Again, the large Pdots in water exhibit fast photobleaching, while the Pdots in OSS maintain relatively stable fluorescence. The average photobleaching percentage of PFBT43 in OSS was determined to be 33\%, about two times lower than the value determined for the same Pdots in water (72\%) (Figure 4(c)). Large sized PFBT43 Pdots in OSS displayed similar photobleaching percentage to those of small sized PFBT21 Pdots. However, large PFBT43 Pdots in water show slightly lower photobleaching percentage (72\%) than small PFBT21 Pdots (86\%). This is likely due to the fact that single-particles of PFBT43 Pdots contain more polymer chromophores than small sized PFBT21 Pdots, therefore a fraction of chromophores are still active after the continuous excitation of 120 seconds. Nevertheless, both PFBT21 and PFBT43 in OSS show substantially improved photostability as compared to the Pdots in water, indicating the important role of OSS in long-term singleparticle imaging and tracking applications. In previous study, the photostability of Pdots was improved by doping with hydrophobic antifade additives [25]. This study provided the enzymatic OSS that can enhance the single-particle brightness about two times and suppress the fast photobleaching component of Pdots in long-term single-particle imaging.

\section{Conclusion}

In summary, we provided an effective enzymatic oxygen scavenging system (OSS) to enhance single-particle brightness and photostability of Pdots. Single-particle imaging showed that the per-particle brightness of PFBT21 Pdot was increased by about two times in the presence of the OSS. We investigated the photostability of the Pdots by photobleaching statistics, which indicated that the PFBT Pdots in OSS showed a substantial improvement in 
photostability as compared to the Pdots in water. Particularly, the OSS efficiently suppressed the fast bleaching component of Pdots. This study provides a useful approach for enhancing single-particle brightness and photostability of Pdots in long term single-particle tracking applications.

\section{Acknowledgements}

This work was supported by "Thousand Young Talents Program" and the key program from National Science Foundation of China (Grant No. 61335001).

\section{References}

[1] S. Weiss, Fluorescence Spectroscopy of Single Biomolecules, Science 283 (1999) 16761683.

[2] G. Seisenberger, M. U. Ried, T. Endress, H. Büning, M. Hallek, and C. Bräuchle, RealTime Single-Molecule Imaging of the Infection Pathway of an Adeno-Associated Virus, Science 294 (2001) 1929-1932.

[3] X. S. Xie, P. J. Choi, G. W. Li, N. K. Lee, and G. Lia, Single-Molecule Approach to Molecular Biology in Living Bacterial Cells, Annu. Rev. Biophys. 37 (2008) 417-444.

[4] M. Goulian, and S. M. Simon, Tracking single proteins within cells, Biophys. J. 79 (2000) 2188-2198.

[5] C. F. Wu, B. Bull, C. Szymanski, K. Christensen, and J. McNeill, Multicolor conjugated polymer dots for biological fluorescence imaging, ACS nano 2 (2008) 2415-2423.

[6] G. W. Li, and X. S. Xie, Central dogma at the single-molecule level in living cells, Nature 475 (2011) 308-315.

[7] J. C. M. Gebhardt, D. M. Suter, R. Roy, Z. W. Zhao, A. R. Chapman, S. Basu, T. Maniatis, and X. S. Xie, Single-molecule imaging of transcription factor binding to DNA in live mammalian cells, Nat. Methods. 10 (2013) 421-426.

[8] Y. Taniguchi, P. J. Choi, G.-W. Li, H. Chen, M. Babu, J. Hearn, A. Emili, and X. S. Xie, Quantifying E. coli proteome and transcriptome with single-molecule sensitivity in single cells, Science 329 (2010) 533-538.

[9] M. Bates, B. Huang, G. T. Dempsey, and X. W. Zhuang, Multicolor super-resolution imaging with photo-switchable fluorescent probes, Science 317 (2007) 1749-1753. 
[10] B. Huang, W. Q. Wang, M. Bates, and X. W. Zhuang, Three-dimensional superresolution imaging by stochastic optical reconstruction microscopy, Science 319 (2008) 810-813.

[11] E. Betzig, G. H. Patterson, R. Sougrat, O. W. Lindwasser, S. Olenych, J. S. Bonifacino, M. W. Davidson, J. L. Schwartz, and H. F. Hess, Imaging intracellular fluorescent proteins at nanometer resolution, Science 313 (2006) 1642-1645.

[12] R. Iino, I. Koyama, and A. Kusumi, Single molecule imaging of green fluorescent proteins in living cells: E-cadherin forms oligomers on the free cell surface, Biophys. J. 80 (2001) 2667-2677.

[13] D. Magde, R. Wong, and P. G. Seybold, Fluorescence quantum yields and their relation to lifetimes of rhodamine $6 \mathrm{G}$ and fluorescein in nine solvents: improved absolute standards for quantum yields, Photochem. Photobiol. 75 (2002) 327-334.

[14] X. Michalet, F. F. Pinaud, L. A. Bentolila, J. M. Tsay, S. Doose, J. J. Li, G. Sundaresan, A. M. Wu, S. S. Gambhir, and S. Weiss, Quantum Dots for Live Cells, in Vivo Imaging, and Diagnostics, Science 307 (2005) 538-544.

[15] C. F. Wu, and D. T. Chiu, Highly fluorescent semiconducting polymer dots for biology and medicine, Angew. Chem. Int. Ed. Engl. 52 (2013) 3086-3109.

[16] K. Y. Pu, A. J. Shuhendler, J. V. Jokerst, J. Mei, S. S. Gambhir, Z. Bao, and J. Rao, Semiconducting polymer nanoparticles as photoacoustic molecular imaging probes in living mice, Nat. nanotechnol. 9 (2014) 233-239.

[17] K. Y. Pu, A. J. Shuhendler, and J. Rao, Semiconducting polymer nanoprobe for in vivo imaging of reactive oxygen and nitrogen species, Angew. Chem. Int. Ed. Engl. 52 (2013) 10325-10329.

[18] H. X. Yuan, B. Wang, F. T. Lv, L. Liu, and S. Wang, Conjugated- Polymer- Based Energy- Transfer Systems for Antimicrobial and Anticancer Applications, Adv. Mater. 26 (2014) 6978-6982.

[19] X. L. Feng, F. T. Lv, L. B. Liu, Q. Yang, S. Wang, and G. C. Bazan, A highly emissive conjugated polyelectrolyte vector for gene delivery and transfection, Adv. Mater. 24 (2012) 5428-5432.

[20] K. Li, D. Ding, D. Huo, K. Y. Pu, N. N. P. Thao, Y. Hu, Z. Li, and B. Liu, Conjugated Polymer Based Nanoparticles as Dual- Modal Probes for Targeted In Vivo Fluorescence and Magnetic Resonance Imaging, Adv. Funct. Mater. 22 (2012) 3107-3115.

[21] J. B. Yu, C. F. Wu, Z. Y. Tian, and J. McNeill, Tracking of single charge carriers in a conjugated polymer nanoparticle, Nano Lett. 12 (2012) 1300-1306. 
[22] Z. H. Liu, Z. Z. Sun, W. H. Di, W. P. Qin, Z. Yuan, and C. F. Wu, Brightness calibrates particle size in single particle fluorescence imaging, Opt. Lett. 40 (2015) 1242-1245.

[23] T. L. Andrew, and T. M. Swager, Reduced photobleaching of conjugated polymer films through small molecule additives, Macromolecules. 41 (2008) 8306-8308.

[24] Q. S. Zheng, S. Jockusch, G. G. Rodríguez-Calero, Z. Zhou, H. Zhao, R. B. Altman, H.D. Abruñab and S.C. Blanchard, Intra-molecular triplet energy transfer is a general approach to improve organic fluorophore photostability, Photochem. Photobiol. Sci. 15, (2016) 196-203.

[25] Z. Y. Tian, J. B. Yu, X. L. Wang, L. C. Groff, J. L. Grimland, and J. D. McNeill, Conjugated polymer nanoparticles incorporating antifade additives for improved brightness and photostability, J. Phys. Chem. B 117 (2012) 4517-4520.

[26] C. F. Wu, C. Szymanski, and J. McNeill, Preparation and encapsulation of highly fluorescent conjugated polymer nanoparticles, Langmuir 22 (2006) 2956-2960.

[27] K. Sun, H. B. Chen, L. Wang, S. Y.Yin, H. Y. Wang, G. X. Xu, D. N. Chen, X. Zhang, C. F. Wu, and W. P. Qin, Size-dependent property and cell labeling of semiconducting polymer dots, ACS Appl. Mater. Interfaces 6 (2014) 10802-10812.

[28] C. F. Wu, T. Schneider, M. Zeigler, J. B. Yu, P. G. Schiro, D. R. Burnham, J. D. McNeill, and D. T. Chiu, Bioconjugation of ultrabright semiconducting polymer dots for specific cellular targeting, J. Am. Chem. Soc. 132 (2010) 15410-15417.

[29] Y. S. Wang, B. Liu, A. Mikhailovsky, and G. C. Bazan, Conjugated polyelectrolyteMetal nanoparticle platforms for optically amplified DNA detection, Adv. Mater. 22 (2010) 656-659.

[30] C. T. Kuo, A. M. Thompson, M. E. Gallina, F. M. Ye, E. S. Johnson, W. Sun, M. X. Zhao, J. Yu, I. C. Wu, and B. Fujimoto, Optical painting and fluorescence activated sorting of single adherent cells labelled with photoswitchable Pdots, Nat. Commun. 7 (2016) 11468.

[31] C. E. Aitken, R. A. Marshall, and J. D. Puglisi, An oxygen scavenging system for improvement of dye stability in single-molecule fluorescence experiments, Biophys. J. 94 (2008) 1826-1835.

[32] P. Wu, Y. He, H. F. Wang, and X. P. Yan, Conjugation of glucose oxidase onto Mndoped $\mathrm{ZnS}$ quantum dots for phosphorescent sensing of glucose in biological fluids, Anal. Chem. 82 (2010) 1427-1433. 
[33] Z. Y. Wang, S. N. Liu, P. Wu, and C. X. Cai, Detection of glucose based on direct electron transfer reaction of glucose oxidase immobilized on highly ordered polyaniline nanotubes, Anal. Chem. 81 (2009) 1638-1645.

[34] J. C. Bolinger, M. C. Traub, T. Adachi, and P. F. Barbara, Ultralong-range polaroninduced quenching of excitons in isolated conjugated polymers, Science 331 (2011) 565567.

[35] Z. P. Zeng, X. Z. Chen, H. N. Wang, N. Huang, C. Y. Shan, H. Zhang, J. L. Teng, and P. $\mathrm{Xi}$, Fast super-resolution imaging with ultra-high labeling density achieved by joint tagging super-resolution optical fluctuation imaging, Sci. Rep. 5 (2015) 08359.

[36] S. Liu, N. J. Borys, S. Sapra, A. Eychmüller, and J. M. Lupton, Localization and Dynamics of Long- Lived Excitations in Colloidal Semiconductor Nanocrystals with Dual Quantum Confinement, Chemphyschem 16 (2015) 1663-1669.

[37] C. F. Wu, C. Szymanski, Z. Cain, and J. McNeill, Conjugated polymer dots for multiphoton fluorescence imaging, J. Am. Chem. Soc. 129 (2007) 12904-12905. 
Figure 1 (a) Chemical structure of semiconductor polymer PFBT and functional polymer PSMA. (b) Dynamic light scattering (DLS) results of PFBT Pdots. (c) Typical transmission electron microscopy (TEM) image of the Pdots. (d) The UV/VIS absorption spectrum and the fluorescence spectrum of the PFBT Pdots.

Figure 2 Single-particle imaging brightness of PFBT Pdots. (a) and (c) show the singleparticle fluorescence images of Pdots in the oxygen scavenging system and the deionized water, respectively. The images were obtained under identical excitation and detection conditions. (b) and (d) show the intensity histograms by analyzing single-particle brightness of hundreds of Pdots. The black curves were obtained by fitting a lognormal distribution to the histogram, resulting in average intensity of 39452 and 20104 counts for the Pdots in OSS and in water, respectively. Scale bar represents $20 \mu \mathrm{m}$.

Figure 3 Five typical photobleaching trajectories of the PFBT21 Pdots in water (a) and in OSS (b), respectively. (c) Single particle photobleaching of PFBT21 Pdots in OSS and water. (d) The bleaching percentage statistics histogram distributions of the PFBT21 Pdots in OSS and in water. The black curves were obtained by fitting a lognormal distribution to the histogram, resulting in average bleaching percentage of $29 \%$ and $86 \%$ for the PFBT21 Pdots in OSS and water, respectively.

Figure 4 Five typical photobleaching trajectories of the PFBT43 Pdots in water (a) and in OSS (b), respectively. (c) The bleaching percentage statistics histogram distributions of the PFBT43 Pdots in OSS and in water. The black curves were obtained by fitting a lognormal distribution to the histogram, resulting in average bleaching percentage of $33 \%$ and $72 \%$ for the PFBT43 Pdots in OSS and water, respectively. 
(a)
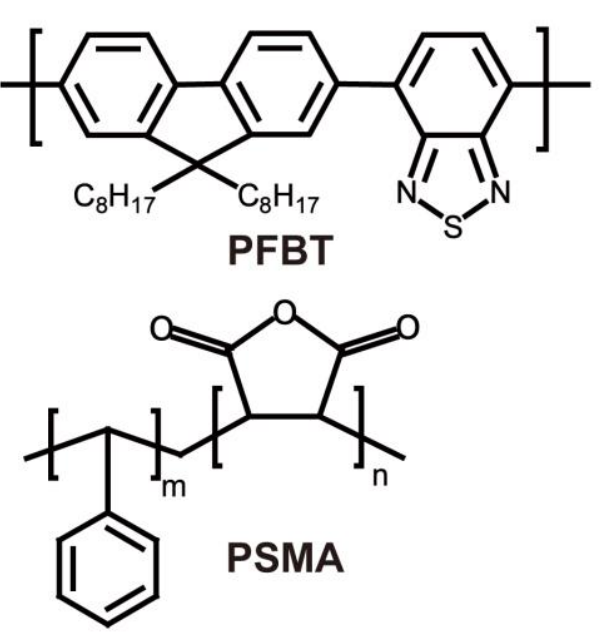

(c)

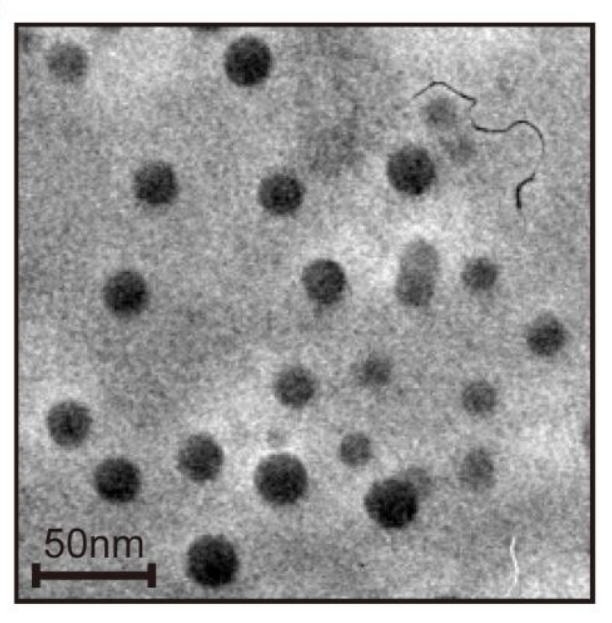

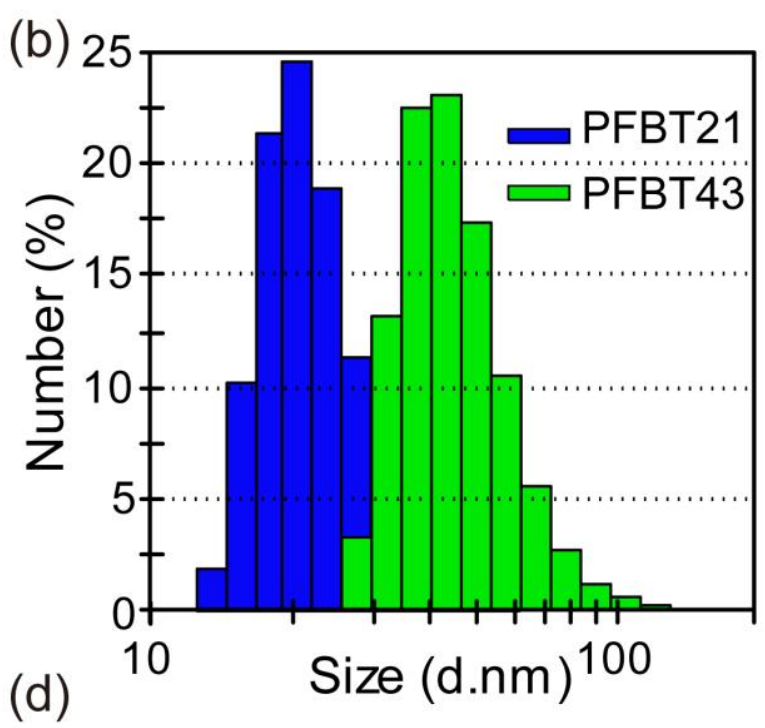

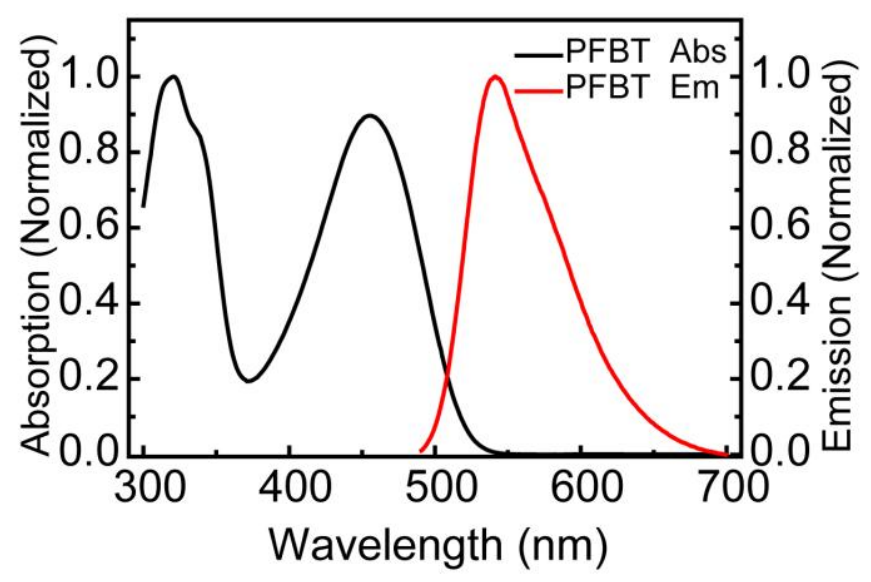




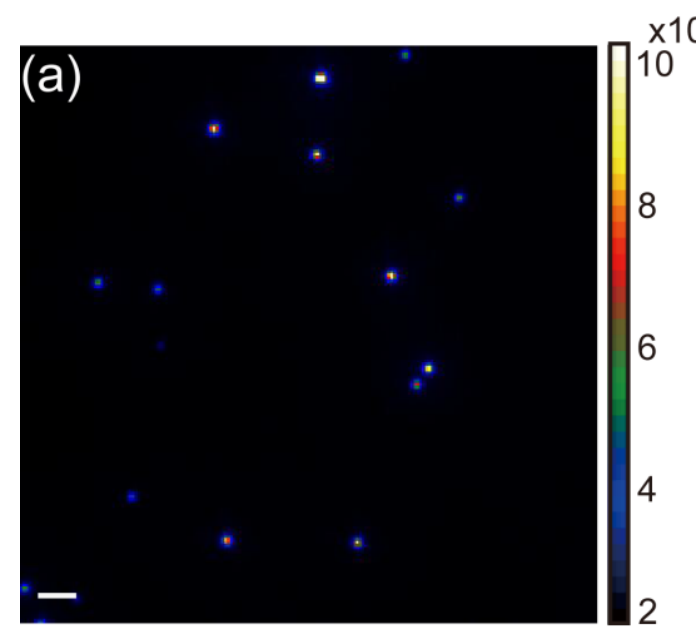

(b)
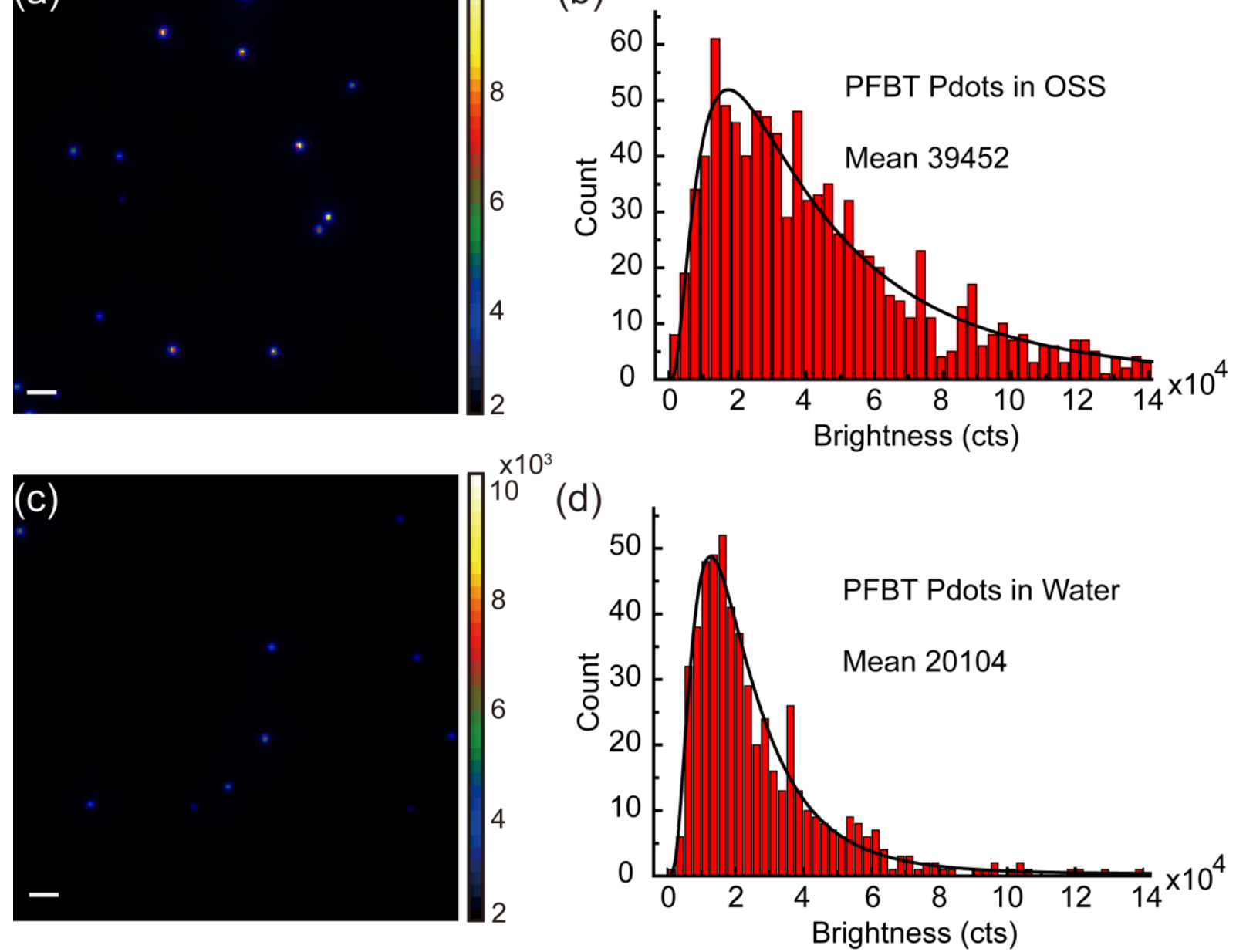
(a)

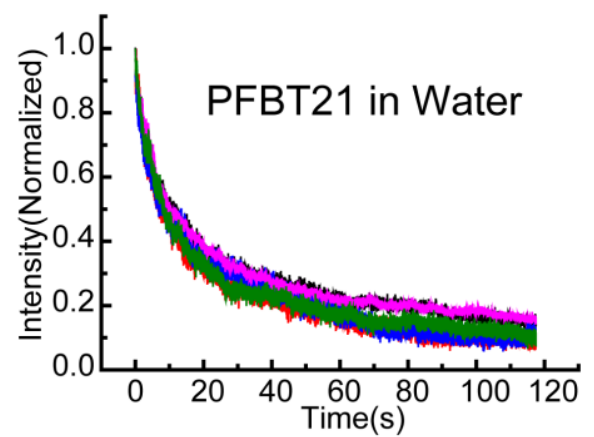

(b)

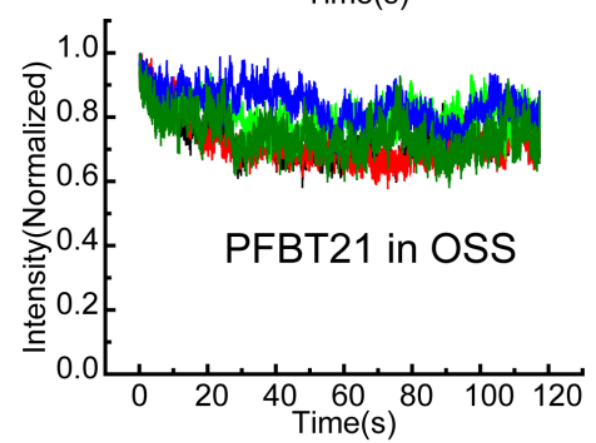

(c)

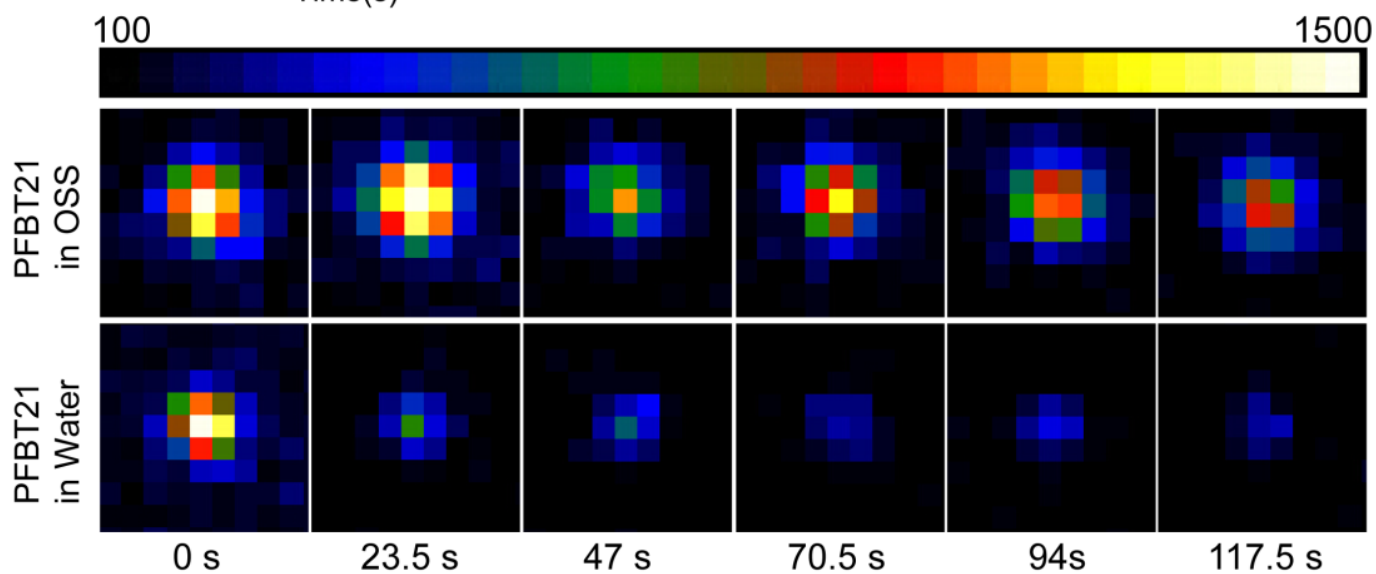

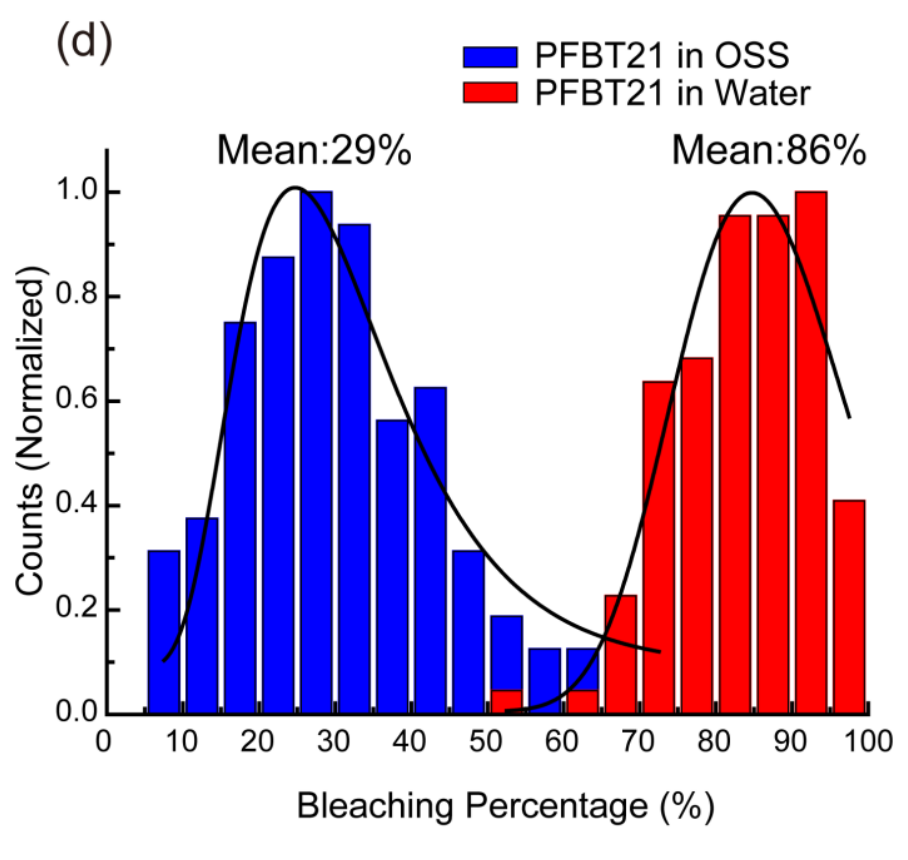

1500 
(a)

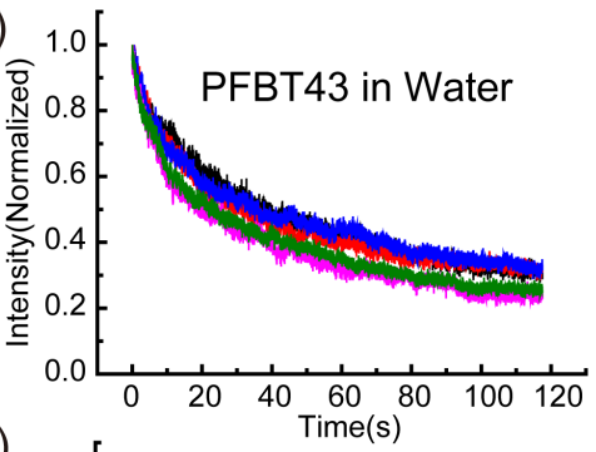

(b)

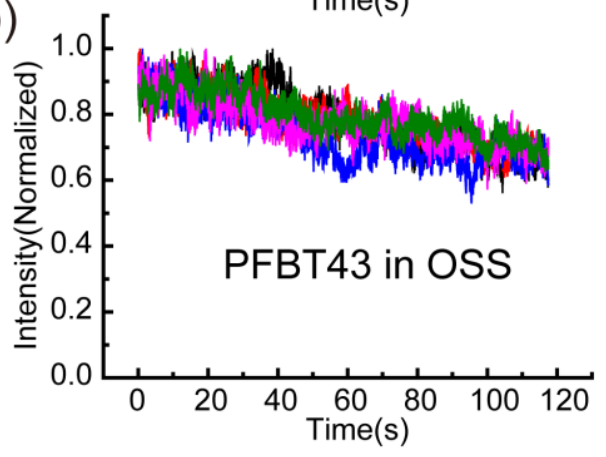

(c) - PFBT43 in OSS $\square$ PFBT43 in Water

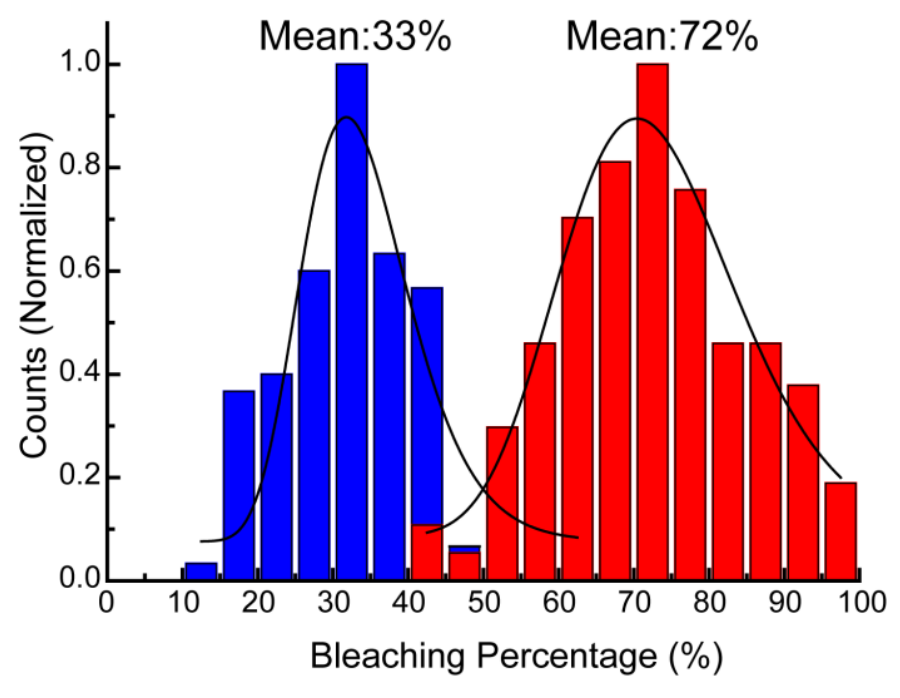


Enhanced single-particle brightness and photostability of semiconductor polymer dots by enzymatic oxygen scavenging system

Zhihe Liu ${ }^{\mathrm{a}}$, Yingkun Yang ${ }^{\mathrm{a}}$, Zezhou Sun ${ }^{\mathrm{a}}$, and Changfeng $\mathrm{Wu}^{\mathrm{a}}$,*

${ }^{\text {a }}$ State Key Laboratory on Integrated Optoelectronics, College of Electronic Science and Engineering, Jilin University, Changchun, Jilin, 130012, China

*Corresponding Author: cwu@jlu.edu.cn
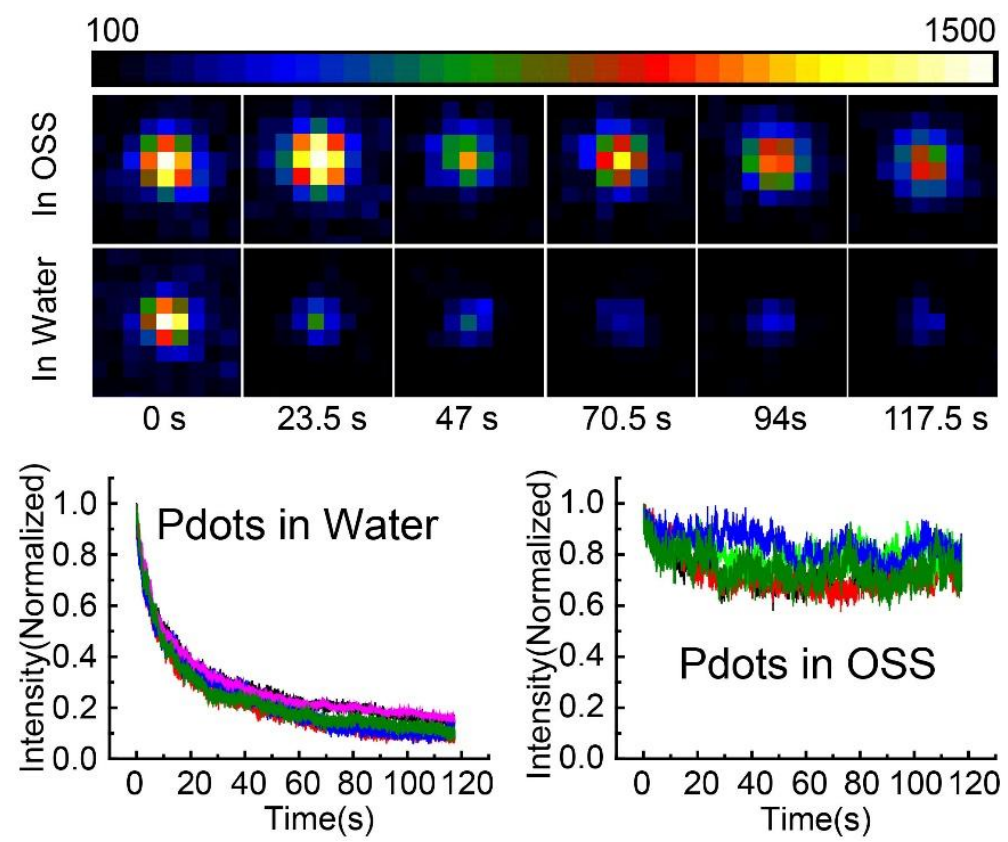

Single-particle fluorescence brightness of polymer dots exhibits a two-fold increase by an enzymatic oxygen scavenging system. The photostability of Pdots in the oxygen scavenging system was significantly improved as compared to those in water, providing a useful approach for enhancing single-particle brightness and photostability in long term imaging and tracking applications. 